\title{
Airway management for microlaryngeal surgery
}

\author{
Marinov Ts.', M. Belitova', T. Popov², N. Nizamova², J. Rangachev², D. Popova ${ }^{2}$ \\ 'Department of Anesthesiology and Intensive Care; \\ University Hospital "Queen Giovanna"- ISUL; Medical University-Sofia \\ 2Department of ENT Surgery; University Hospital "Queen Giovanna" - ISUL; \\ Medical University - Sofia
}

\begin{abstract}
Theoretical basis: Microlaryngeal surgery can be conducted in an awake patient, frequently under conscious sedation, or with the patient anesthetized. The ventilation options under general anesthesia consist of "tube" (i.e., endotracheal intubation) and "tubeless" techniques, with the latter represented by the techniques of Spontaneous ventilation, Apneic intermittent ventilation (AIV), and Jet Ventilation (JV). Results: The use of a small (5.0-mm ID) MLT tube with positive-pressure ventilation remains the standard for airway management in most nonlaser microlaryngeal surgery, and it is associated with minimal or no intraoperative complications. Spontaneous ventilation is rarely used in adult microlaryngeal surgery, but it is commonly employed in the pediatric patient population. AIV remains a relatively popular technique for microlaryngeal surgical procedures of short duration in some surgical centers. Superimposed High-Frequency Jet Ventilation (SHFJV), which combines highfrequency and low-frequency ventilation modes, has been used effectively in surgical treatment of high-grade laryngeal and tracheal stenosis. Conclusion: General anesthesia for microlaryngeal surgery represents a unique example of close cooperation between the surgeon and the anesthesiologist.
\end{abstract}

Key words: Microlaryngeal surgery, general anesthesia, ventilation techniques.

\section{Literature review}

Microlaryngeal surgery can be conducted in an awake patient, frequently under conscious sedation, or with the patient anesthetized. The ventilation options under general anesthesia consist of "tube" (i.e., endotracheal intubation) and "tubeless" techniques, with the latter represented by the techniques of Spontaneous ventilation, Apneic intermittent ventilation (AIV), and Jet Ventilation $(\mathrm{JV}){ }^{1,2}$

\section{Awake airway surgery}

For selected patients, many laryngoscopic procedures can be safely and effectively performed in an office-based setting, including diagnostic endoscopy, laser surgery, microlaryngeal surgery for cancer screening and biopsies, and therapeutic vocal cord injections. The key to success for office-based surgery remains adequate topical and regional anesthesia of the patient's airway, which is usually performed by the surgeon and typically follows preparation of the patient for awake oral and nasal flexible fiberoptic intubation. Although highly motivated patients can undergo office-based laryngoscopic surgery strictly under local anesthesia, most desire sedation and amnesia.

If presence of the anesthesiologist is requested, the main objectives are to monitor for possible local anesthetic toxicity, to supplement local anesthesia with a rapidly titratable and reversible state of sedation, and to treat acute hyperdynamic respons- 
es that can occur in up to $20 \%$ to $30 \%$ of patients, despite seemingly adequate topical anesthesia of the airway. ${ }^{3}$ Judicious use of intravenous opioids or sedatives/hypnotics, or both, is paramount, because a loss of patient cooperation may result in intraoperative injury. Sedation of the patients with obstructive sleep apnea and morbid obesity should be performed with extreme caution. ${ }^{4,5}$

\section{Asleep airway surgery}

General anesthesia for microlaryngeal surgery represents a unique example of some of the conflicting intraoperative goals that exist between the surgeon and the anesthesiologist with regard to the patient's airway control and maintenance. For the surgeon, ideal operating conditions would be completely unobstructed surgical visualization, unimpeded surgical manipulation, and absence of movement in the surgical field. From the anesthesiologist's perspective, the ideal anesthetic technique would allow adequate protection of the patient's lower airway from aspiration and the use of stable, controlled mechanical ventilation with the ability to measure the concentration of anesthetic gases, peak inspiratory pressure (PIP), inspired oxygen concentration $\left(\mathrm{FiO}_{2}\right)$, and end-tidal carbon dioxide level $\left(\mathrm{EtCO}_{2}\right)$. In most cases, these objectives can be balanced by the use of a small microlaryngeal tracheal tube (MLT tube), maximizing the patient's safety and the success of surgery.

\section{Endotracheal intubation with microlaryngeal tracheal tubes}

The use of a small (5.0-mm ID) MLT tube with positive-pressure ventilation remains the standard for airway management in most nonlaser microlaryngeal surgery, and it is associated with minimal or no intraoperative complications. ${ }^{6,7}$ Adequate gas exchange can be maintained through small-ID ETTs in most adult patients, unless the duration of surgery approaches 2 hours. Even then, despite a consistent trend toward progressive hypercapnia and respiratory acidosis, the $\mathrm{pH}$ and $\mathrm{EtCO} 2$ values remain within physiologic range. ${ }^{8,9}$

With most glottic pathology originating in the anterior two thirds of the larynx, consistent positioning of a small MLT tube between the arytenoid cartilages in the posterior part of the glottis leaves most of the surgical field unobstructed to the surgical view and manipulations. Even with many posterior glottic disorders, it may be possible for the surgeon to gently displace the MLT tube anteriorly with the microsurgical cupped forceps or to perform the surgery using the specially designed posterior glottic laryngoscopes. ${ }^{10,11,12}$

However, if the posterior glottis is occupied by a significant surgical pathology (e.g., posterior glottic or subglottic stenosis, transglottic tumor), use of alternative, tubeless ventilation techniques becomes necessary. Because of the surgeon's preference, tubeless ventilation can also be requested as a primary ventilation mode from the outset of the procedure.

\section{Tubeless techniques Spontaneous Ventilation}

Spontaneous ventilation is rarely used in adult microlaryngeal surgery, but it is commonly employed in the pediatric patient population, for whom it offers the additional ability to evaluate dynamic airway function and the level of obstruction. ${ }^{13,14}$ Although this technique offers free access to the larynx, it does not provide a still surgical field for precision surgery, it affords no protection of the lower airway, and it contaminates the OR environment. Deep planes of anesthesia are usually required to blunt the laryngeal responses and to prevent patient movement, which tends to provoke cardiovascular instability and ventilatory compromise (i.e., hypoxemia, hypercarbia, and short periods of apnea). ${ }^{15}$ The protagonists of spontaneous ventilation technique may wish to routinely supplement general anesthesia with topical or local anesthesia of the airway (usually done by the surgeon after deployment of suspension laryngoscopy), which facilitates maintenance of a more stable and lighter plane of anesthesia, promotes hemodynamic and respiratory stability, and decreases the incidence of intraoperative laryngospasm. ${ }^{16}$

\section{Apneic intermittent ventilation}

AIV remains a relatively popular technique for microlaryngeal surgical procedures of short duration in some surgical centers. Compared with spontaneous ventilation, it affords more stable and controlled anesthetic conditions, as well as full muscle relaxation. After induction of anesthesia, the patient's lungs are ventilated by a face mask or an LMA, which is followed by a period of apnea to allow deployment of a suspension laryngoscope by the surgeon. The patient's trachea is subsequently intubated by the surgeon with a small-diameter, 
preferably uncuffed ETT that is placed through the lumen of the laryngoscope, and the patient's lungs are hyperventilated with an $\mathrm{FiO}_{2}$ of 1.0. The ETT is then removed to provide a fully unobstructed and still surgical view of the larynx. The ETT is withdrawn and reinserted as frequently as necessary to maintain an oxygen saturation by pulse oximetry $\left(\mathrm{SpO}_{2}\right)$ of $90 \%$ or greater and $\mathrm{EtCO}_{2}$ between 40 and $60 \mathrm{~mm} \mathrm{Hg}$, allowing periods of apnea up to 5 to 10 minutes in healthy adult patients. ${ }^{17,18}$ TIVA is typically used for maintenance. Monitoring the hypnotic state of anesthesia is advisable during AIV, because the incidence of awareness and recall may reach 4\% (30 times higher than in the general surgical population), especially when the inhalational agents are used to supplement intravenous anesthesia. ${ }^{19,20}$

The disadvantages of AIV include slowing the pace of surgery, disruption of the surgical field, possible trauma to the vocal cords and lower airway due to repeated endotracheal intubation, and a propensity for laryngospasm. In a study of more than 350 patients, the incidence of intraoperative laryngospasm with AIV was $1.4 \%$. The AIV may not be suitable for patients with significant lung or cardiovascular disease, and it leaves the patient's lower airway unprotected to aspiration.

\section{Jet Ventilation}

Supraglottic JV (i.e., jet nozzle above the glottic opening) for microlaryngeal surgery can be performed through the side port of a suspension operating laryngoscope, with the jet cannula attached to the lumen of the laryngoscope or through a specialized jet laryngoscope. ${ }^{21,22,23}$

Subglottic JV (i.e., jet nozzle below the glottic opening) is established by bypassing the larynx from above (i.e., translaryngeal or transglottal approach) or below (i.e., percutaneous approach) through the Cricotracheal membrane (CTM) or the upper Transtracheal Jet Ventilation (TTJV) rings. Transglottal JV typically employs specialized, laser-safe, small-diameter, orally placed, double-lumen catheters, in which the large port is used for jetting and the smaller lumen for monitoring the distal airway pressure and respiratory gases. ${ }^{24,25}$ Long, single-lumen catheters (typically 1.5- to 3-mm ID), some of which are laser resistant, may be used and can be placed through the oral or nasal route, however, they lack concurrent monitoring capability. ${ }^{26,27,28}$ Alternatively, a small-diameter, movable, metal jet cannula can be passed through the glottis by the surgeon after the suspension laryngoscope is in position..$^{29}$ For transglottal JV, midtracheal placement of the catheter or cannula is usually preferred. TTJV is typically administered through a long catheter or Ravussin-type cannula. ${ }^{30}$ For TTJV catheter or cannula placement, the use of an flexible fiberoptic bronchoscope (FFB) or a rigid bronchoscope may be advocated to monitor the procedure and to minimize the risk of unnoticed posterior tracheal wall laceration, which may lead to submucosal gas injection and barotrauma. ${ }^{31}$ Use of a rigid bronchoscope with the bevel turned posteriorly may be especially efficacious, because the posterior tracheal wall is protected by the bronchoscope from the needle entry. For transglottal JV and TTJV, endoscopic control also allows adjustment of the position of the distal end of the catheter or cannula to optimize high frequency jet ventilation (HFJV).

Compared with endotracheal intubation, supraglottic and subglottic JV techniques have distinct advantages of providing the surgeon with an enlarged, clear or minimally impeded, and undistorted view of the endolarynx and facilitating surgical access. Although supraglottic and subglottic ventilation techniques can use low-frequency jet ventilation (LFJV), HFJV, or superimposed high-frequency jet ventilation (SHFJV) modes, the use of these modes in clinical practice is usually more restrictive. ${ }^{32,33,34}$

The use of manual supraglottic LFJV (i.e., Venturi jet ventilation) at a rate of less than 60 breaths/min continues to predominate in clinical practice, probably because of the low cost and easy accessibility of manual JV devices. ${ }^{35,36}$ Although an overall incidence of complications with manual supraglottic LFJV may be low $(0.42 \%)$, a survey of 229 U.K. centers revealed that it was responsible for most major complications (e.g., significant hypoxemia, barotrauma, unplanned admission to the intensive care unit) and for all deaths, especially when applied subglottically. This suggests that LFJV should be reserved for uncomplicated, elective procedures of short duration and that it may not be regarded as a standard of practice for microlaryngeal surgery.

The subglottic HFJV mode (respiratory rate of 100 to 300 breaths/min; tidal volumes [Vt] of 1 to 3 $\mathrm{mL} / \mathrm{kg}$ ), delivered through specialized automated jet ventilators, is typically used. Compared with 
supraglottic LFJV, in which intermittent apnea is frequently required due to significant vocal cord movement, subglottic HFJV significantly reduces laryngeal motion and affords a quiet surgical field without the need for interrupting ventilation. If vocal cord movement becomes a problem, HFJV driving pressure can be decreased, and the respiratory frequency can be increased to provide a smoother gas flow, or the ventilator can be turned off during particularly delicate parts of the procedure. ${ }^{34}$

Despite very small Vt values, $\mathrm{CO}_{2}$ elimination during subglottic HFJV is facilitated by the upstream turbulent convective flow of $\mathrm{CO}_{2}$ along the decreasing gradient from the alveoli to the conducting airways. The alveolar-arterial $\mathrm{CO}_{2}$ gradient in patients with normal lung function is largely maintained within normal range. ${ }^{37}$

In contrast to supraglottic LFJV, with which contamination of the lower airway due to air entrainment is possible, a continuous, upward-directed flow of gas during subglottic HFJV creates a positive-pressure build-up, preventing blood and surgical debris from being directed down an unprotected airway. Alternatively, initiation of the subglottic HFJV can be held off until the suspension laryngoscope is deployed, and ventilation is supported conventionally through a face mask or the LMA.

On emergence from anesthesia, small Vt values and low peak and mean airway pressures associated with subglottic HFJV enable the patient to breathe spontaneously, facilitating a transition to adequate spontaneous ventilation. ${ }^{38,39}$ This transition can be further assisted at the end of surgery by increasing the frequency of ventilation to 300 breaths $/ \mathrm{min}$, increasing $\mathrm{FiO}_{2}$ to 1.0 , and setting a ventilator driving pressure at about 0.8 bar, which enables almost continuous flow of $\mathrm{O}_{2}$ and apneic oxygenation, as well as a rise in the carbon dioxide $\left(\mathrm{CO}_{2}\right)$ level. If the conversion to spontaneous ventilation through a small subglottic catheter proves difficult, the patient's airway can be supported through a face mask, LMA, or ETT, as required. If obstructive airway lesions exist, subglottic HFJV must be used with extreme caution. If upper airway obstruction is greater than $50 \%$, the position of the jet nozzle should be proximal to the site of the obstruction to prevent barotrauma, or the obstruction must be bypassed by a rigid bronchoscope first.

Total outflow obstruction with resultant barotrauma during subglottic HFJV can be quickly precipitated by or closure of the vocal cords due to inadequate depth of anesthesia or inadequate muscle relaxation. Modern automated jet ventilators incorporate multiple safety features, including automatic ventilator shutdown, if the user-preset pressure limits are exceeded. This design has enabled some experienced providers to successfully use high-frequency TTJV in patients with massive supraglottic lesions and severe airway compromise, for which the use of supraglottic or subglottic JV was not possible or surgically feasible. The presence of a second anesthesiologist to facilitate monitoring and maintenance of an upper airway was required and deemed an important safety factor in preventing intraoperative pressure-related complications in all cases.

Compared with the transglottal approach, high-frequency TTJV is associated with a significantly higher combined major and minor (e.g., transient hypoxemia) complication rate and it represents an independent risk factor for complications during $\mathrm{JV}$ for microlaryngeal surgery. Modern automated JV may not be able to remediate all possible causes of barotrauma associated with high-frequency TTJV; complications may be related to the TTJV catheter insertion problems, laryngospasm, and high-pressure episodes (e.g., coughing, active expiration) during the recovery period.

SHFJV, which combines high-frequency and low-frequency ventilation modes, has been used effectively in surgical treatment of high-grade laryngeal and tracheal stenosis, even with a remaining glottic opening as small as 2 to $3 \mathrm{~mm} .{ }^{40} \mathrm{SHFJV}$ is delivered supraglottically through a specialized jet laryngoscope, which incorporates welded low-frequency and high-frequency jet nozzles. ${ }^{41} \mathrm{As}$ the streams (LFJV of 12 to 20 breaths/min; HFJV of 100 to 900 breaths/min) get simultaneously directed from the ventilator toward the center of the distal end of the jet laryngoscope, LFJV entrains air and produces cyclic changes in $\mathrm{Vt}$ (similar to supraglottic LFJV), facilitating maintenance of $\mathrm{PaCO}_{2}$ at near-normal limits and allowing HFJV to be adjusted as needed. HFJV builds up a continuous PEEP and promotes alveolar recruitment, maintaining $\mathrm{PaCO}_{2}$ even in the presence of the low $\mathrm{FiO}_{2}$ required for laser surgery. ${ }^{42,43}$ Safety of SHFJV is enhanced by an integrated port for continuous pressure (PIP and PEEP) and gas ( $\mathrm{FiO} 2$ and $\mathrm{EtCO}_{2}$ ) monitoring at the end of the jet laryngoscope and of an automatic pressure-triggered 
ventilator shutdown feature, similar to an isolated HFJV mode.

To achieve adequate SHFJV, it appears to be sufficient to generate a PIP of 15 to $30 \mathrm{~cm} \mathrm{H} \mathrm{H}_{2}$, as measured at the end of the jet laryngoscope, which closely correlates with the PIP at the glottic and tracheal levels (i.e., no further increase in pressure occurs in the distal airway). The PEEP values may not exceed 2.5 to $5 \mathrm{~cm} \mathrm{H}_{2} \mathrm{O}$. As a result, no adverse hemodynamic effects and barotrauma were observed in more than 1500 adult and pediatric patients who had undergone supraglottic SHFJV for laryngotracheal surgery, and endotracheal intubation was required in only 3 patients $(0.2 \%)$, with concomitant significant restrictive or obstructive pulmonary disease. Due to the HFJV component, vocal cord movement is greatly attenuated during SHFJV. If a perfectly still surgical field is requested by the surgeon, HFJV can be further increased, LFJV decreased or stopped, or a short period of full apnea instituted..$^{40,41}$
SHFJV is a completely tubeless, laser-safe, open breathing system that allows a fully unobstructed surgical field. It enables an easy switch between different JV modes and parameters, and it offers greater versatility and ventilation capabilities over the single-frequency JV techniques, especially in patients with preexisting compromised gas exchange. However, its effective use requires optimal laryngoscope alignment and adjustability in relation to the glottic opening.

Despite the increased safety profile of SHFJV, clinical monitoring of the patient to prevent barotrauma should remain the standard of care for all JV techniques. Close cooperation between the surgeon and the anesthesiologist is essential. If the operating laryngoscope moves or is removed and obstructs the airway without a warning to the anesthesia team, major barotrauma may result. Ensuring an adequate level of anesthesia, analgesia, muscle relaxation and close monitoring of vital signs and chest excursions are essential for the patient's safety.

\section{References}

1. Brooker CR, Hunsaker DH, Zimmerman AA: A new anesthetic system for microlaryngeal surgery. Otolaryngol Head Neck Surg 118:55-60, 1998.

2. Ku PK, Tong MC, Kwan A, van Hasselt CA: Modified tubeless anesthesia during endoscopy for assessment of head and neck cancers. Ear Nose Throat J 82:121-125, 2003.

3. Yung KC, Courey MS: The effect of office-based flexible endoscopic surgery on hemodynamic stability. Laryngoscope 120:2231-2236, 2010.

4. Drummond GB: Comparison of sedation with midazolam and ketamine: Effects on airway muscle activity. Br J Anaesth 76:663-667, 1996.

5. Hillman DR, Platt PR, Eastwood PR: Anesthesia, sleep, and upper airway collapsibility. Anesthesiol Clin 28:443-455, 2010.

6. Bourgain JL, Desruennes E, Fischler M, et al: Transtracheal high frequency jet ventilation for endoscopic airway surgery: A multicentre study. Br J Anaesth 87:870-875, 2001.

7. Jaquet Y, Monnier P, Van Melle G, et al: Complications of different ventilation strategies in endoscopic laryngeal surgery: A 10-year review. Anesthesiology 104:52-59, 2006.

8. Cinar SO, Coskun BU, Cinar U, et al: Blood gas changes in patients undergoing laryngeal microsurgery. Auris Nasus Larynx 33:299-302, 2006.

9. Nicelli E, Gemma M, De Vitis A, et al: Feasibility of standard mechanical ventilation with low Fio2 and small endotracheal tubes during laser microlaryngeal surgery. Head Neck 32:204-209, 2010.

10. Kawaida M, Fukuda H, Kohno N: Microlaryngosurgery for benign posterior glottal lesions using a posterior glottis direct laryngoscope. ORL J Otorhinolaryngol Relat Spec 63:127-130, 2001.

11. Strong MS: Microscopic laryngoscopy. A review and appraisal. Laryngoscope 80:1540-1552, 1970.

12. Ossoff RH, Duncavage JA: Adult subglottiscope for laser surgery. Ann Otol Rhinol Laryngol 97(Pt 1):552-553, 1988.

13. Talmage EA: Safe combined general and topical anesthesia for laryngoscopy and bronchoscopy. South Med J 66:455-459, 1973.

14. Judelman H: Anaesthesia for laryngoscopy and microsurgery of the larynx. S Afr Med J 55:698, 1979.

15. Sun KO: Doxapram in tubeless anaesthesia for microlaryngeal surgery. Anaesth Intensive Care 21:250-251, 1993.

16. Weigand H: Circulatory response to endolaryngeal microsurgery under light general anesthesia and the influence of surface anes $\neg$ thesia. Anesth Analg 48:953-957, 1969.

17. Weisberger EC, Emhardt JD: Apneic anesthesia with intermittent ventilation for microsurgery of the upper airway. Laryngoscope 106:10991102,1996

18. Nelson RA, Miller T: Apneic anesthesia for microlaryngeal surgery. Laryngoscope 83:1228-1233, 1973.

19. Barr AM, Wong RM: Awareness during general anaesthesia for bronchoscopy and laryngoscopy using the apnoeic oxygenation technique. Br J Anaesth 45:894-900, 1973.

20. Sebel PS, Bowdle TA, Ghoneim MM, et al: The incidence of awareness during anesthesia: A multicenter United States study. Anesth Analg 99:833-839, 2004

21. Albert SN, Shibuya J, Albert CA: Ventilation with an oxygen injector for suspension laryngoscopy under general anesthesia. Anesth Analg 51:866-870, 1972 .

22. Aloy A, Schachner M, Cancura W: Tubeless translaryngeal super-imposed jet ventilation. Eur Arch Otorhinolaryngol 248:475-478, 1991.

23. Grasl MC, Donner A, Schragl E, Aloy A: Tubeless laryngotracheal surgery in infants and children via jet ventilation laryngoscope. Laryngoscope 107:277-281, 1997. 
24. Orloff LA, Parhizkar N, Ortiz E: The Hunsaker Mon-Jet ventila $\neg$ tion tube for microlaryngeal surgery: Optimal laryngeal exposure. Ear Nose Throat J 81:390-394, 2002.

25. Barakate M, Maver E, Wotherspoon G, et al: Anaesthesia for microlaryngeal and laser laryngeal surgery: Impact of subglottic jet ventilation. J Laryngol Otol 124:641-645, 2010.

26. Hautmann H, Gamarra F, Henke M, et al: High frequency jet ventilation in interventional fiberoptic bronchoscopy. Anesth Analg 90:14361440,2000

27. Young SS, Wang SJ, Lin SY, et al: An urgent technique of applying high frequency jet ventilation in patients with extreme periglottic stenosis. Acta Anaesthesiol Sin 33:63-68, 1995.

28. Borg U, Eriksson I, Sjöstrand U: High-frequency positive pressure ventilation (HFPPV): A review based upon its use during bron $\neg$ choscopy and for laryngoscopy and microlaryngeal surgery under general anesthesia. Anesth Analg 59:594-603, 1980.

29. Shikowitz MJ, Abramson AL, Liberatore L: Endolaryngeal jet ven-tilation: A 10-year review. Laryngoscope 101:455-461, 1991.

30. Ravussin P, Freeman J: A new transtracheal catheter for ventilation and resuscitation. Can Anaesth Soc J 32:60-64, 1985.

31. Gerig HJ, Heidegger T, Ulrich B, et al: Fiberoptically guided insertion of transtracheal catheters. Anesth Analg 93:663-666, 2001.

32. Vourch G, Fischler M, Michon F, et al: High frequency jet ventila -tion v. manual jet ventilation during bronchoscopy in patients with tracheobronchial stenosis. Br J Anaesth 55:969-972, 1983.

33. Bacher A, Lang T, Weber J, et al: Respiratory efficacy of subglottic low-frequency, subglottic combined-frequency, and supraglottic combinedfrequency jet ventilation during microlaryngeal surgery. Anesth Analg 91:1506-1512, 2000.

34. Patel A, Rubin JS: The difficult airway: The use of subglottic jet ventilation for laryngeal surgery. Logoped Phoniatr Vocol 33:22-24, 2008.

35. Sanders RD: Two ventilating attachments for bronchoscopes. Del Med J 192:170-175, 1967.

36. Gaitini LA, Fradis M, Vaida SJ, et al: Pneumomediastinum due to Venturi jet ventilation used during microlaryngeal surgery in a previously neck-irradiated patient. Ann Otol Rhinol Laryngol 109:519-521, 2000.

37. Bourgain JL, McGee K, Cosset MF, et al: Carbon dioxide monitor $\neg$ ing during high frequency jet ventilation for direct laryngoscopy. Br J Anaesth 64:327-330, 1990 .

38. Young JD, Sykes MK: Assisted ventilation. 1. Artificial ventilation: history, equipment and techniques. Thorax 45:753-758, 1990.

39. Ng A, Russell WC, Harvey N, et al: Comparing methods of admin $\neg$ istering high-frequency jet ventilation in a model of laryngotra $\neg$ cheal stenosis. Anesth Analg 95:764-769, 2002.

40. Rezaie-Majd A, Bigenzahn W, Denk DM, et al: Superimposed high-frequency jet ventilation (SHFJV) for endoscopic laryngo $\neg$ tracheal surgery in more than 1500 patients. Br J Anaesth 96:650-659, 2006.

41. Lanzenberger-Schragl E, Donner A, Grasl MC, et al: Superim $\neg$ posed high-frequency jet ventilation for laryngeal and tracheal surgery. Arch Otolaryngol Head Neck Surg 126:40-44, 2000.

42. Ihra G, Hieber C, Adel S, et al: Tubeless combined high-frequency jet ventilation for laryngotracheal laser surgery in paediatric anaes $\neg$ thesia. Acta Anaesthesiol Scand 44:475-479, 2000.

43. Kraincuk P, Körmöczi G, Prokop M, et al: Alveolar recruitment of atelectasis under combined high-frequency jet ventilation: A com $\neg$ puted tomography study. Intensive Care Med 29:1265-1272, 2003.

\section{Correspondence to:}

Tsvetomir Marinov, MD, PhD

Department of Anesthesiology and Intensive Care

University Hospital "Queen Giovanna”- ISUL

Byalo More Str 8

Medical University-Sofia

E-mail: ts.marinov@abv.bg 